\title{
Reasonable Tag-Based Collaborative Filtering For Social Tagging Systems
}

\author{
Reyn Y. Nakamoto \\ kizasi Company, Inc. \\ Nihonbashi Hakozaki Building \\ 3F \\ Hakozaki 24-1, Nihonbashi \\ Tokyo, Japan 103-0015 \\ reyn@ kizasi.jp \\ Shunsuke Uemura \\ Nara Sangyo University \\ Tateno-Kita 3-12-1, Sango, \\ Ikoma \\ Nara, Japan, 636-8503 \\ uemurashunsuke@nara.su.ac.jp
}

\author{
Shinsuke Nakajima \\ Kyoto Sangyo University \\ Motoyama, Kamigamo, Kita \\ Kyoto, Japan, 603-8555 \\ nakajima@cse.kyoto-su.ac.jp \\ Hirokazu Kato \\ Nara Institute of Science and \\ Technology \\ Takayama 8916-5, Ikoma \\ Nara, Japan, 630-0192 \\ kato@ is.naist.jp
}

\author{
Jun Miyazaki \\ Nara Institute of Science and \\ Technology \\ Takayama 8916-5, Ikoma \\ Nara, Japan, 630-0192 \\ miyazaki@is.naist.jp
}

\author{
Youichi Inagaki \\ kizasi Company, Inc. \\ Nihonbashi Hakozaki Building \\ $3 \mathrm{~F}$ \\ Hakozaki 24-1, Nihonbashi \\ Tokyo, Japan 103-0015 \\ inagaki@ kizasi.jp
}

\begin{abstract}
In this paper, we present a tag-based collaborative filtering recommendation method for use with recently popular online social tagging systems. Combining the information provided by tagging systems with the effective recommendation abilities given by collaborative filtering, we provide a website recommendation system which provides relevant, credible recommendations that match the user's changing interests as well as the user's bookmarking profile. Based upon user testing, our system provides a higher level of relevant recommendations over other commonly used search and recommendation methods. We describe this system as well as the relevant user testing results and its implication towards use in online social tagging systems.
\end{abstract}

\section{Categories and Subject Descriptors}

H.4.m [Information Systems Applications]: Miscellaneous

\section{General Terms}

Algorithms, Experimentation

\section{Keywords}

collaborative filtering, social tagging, recommendation systems

Permission to make digital or hard copies of all or part of this work for personal or classroom use is granted without fee provided that copies are not made or distributed for profit or commercial advantage and that copies bear this notice and the full citation on the first page. To copy otherwise, to republish, to post on servers or to redistribute to lists, requires prior specific permission and/or a fee.

WICOW'08, October 30, 2008, Napa Valley, California, USA.

Copyright 2008 ACM 978-1-60558-259-7/08/10 ...\$5.00.

\section{INTRODUCTION}

As the Internet continues to mature and becomes more accessible to the common user, the amount of information available increases exponentially. Accordingly, finding useful and relevant information is becoming progressively difficult. Moreover, a lot of the information available-blogs, various types of reviews, and so forth-are highly subjective and thus, hard to evaluate purely through content-based algorithms.

For such subjective sources, one person may enjoy something while the next may dislike the same-everyone has differing opinions on what is good. In these cases, people-more so than the current ability of content-based algorithms-are greatly effective in evaluating and filtering this information.

For this reason, Tag-based Contextual Collaborative Filtering (TCCF) was proposed and described in [8]. It was found to be effective in providing website recommendations for social bookmarking systems [7]. This method combines the strengths of both Collaborative Filtering (CF) as well as tagging information from social bookmarking systems to provide effective, personalized recommendations to the user.

By using CF techniques, we can match users with similar preferences. By employing tagging, we can match only the users that liked the same information for the same reasons. Both methods employ users to organize and evaluate information, making them a good fit for each other. With strong user involvement, there is higher confidence in the credibility as well as the quality of the information recommendations.

We now extend our algorithm one step further to provide implicit recommendations by considering the user's current interest. Our new method-called Reasonable Tag-Based Collaborative Filtering or RCF-takes into account the user's bookmarking profile and the currently viewed page to provide relevant website recommendations. We assume that these tags are synonymous with the reasons why they liked something, and thus, we derived the name for our algorithm, Reasonable Tag-Based Collaborative Filtering.

In this paper, we explore the effectiveness of our algorithm and perform user testing as well. In comparison to the other recommendation methods we tested, our method was found 
to be the most effective for implicit live-updating recommendations. We will describe these results and its corresponding implications.

\section{RELATED WORK}

\subsection{Collaborative Filtering Systems}

Collaborative Filtering $(\mathrm{CF})$ is a process that uses the community of users to sort out relevant or important information from the non-relevant and non-important information. The process is based upon the idea that users should enjoy the same items that their similar users like. From a wider perspective, it is the idea of collaborating with other people and sharing knowledge to filter out the best information from the rest. Lastly, CF provides a high level of information credibility since several users are evaluating and rating such information.

$\mathrm{CF}$ has been proven to work well under certain domainsmainly entertainment domains-such as usenet recommendations [9], movie recommendations [5], product recommendations [1], and so forth. Many CF systems rely upon a matrix of numerical ratings on resources by users [9]. Once enough ratings are in place, similarity scores between users are calculated. Based upon these similarity scores, score prediction is then calculated using the similar users' ratings on other items. Those resources with a score prediction above a certain threshold are recommended.

The main drawback of CF is that it only considers if and how much a user likes something. However, it does not take into account the reasons why a user likes something.

\subsection{Social Tagging Systems}

Tagging has been around for sometime, albeit known by other terms such as metadata, categorization, labels, and so forth. Tagging is the process of attaching natural language words as metadata to describe some resource like a movie, photo, book, etc. Tagging vocabulary can be controlled or uncontrolled depending on the type and purpose of the application.

In recent years, the advent of Social Tagging Systems has brought tagging back into the limelight. Currently, there are several popular online social tagging systems which are the subject of continuing research: they range from website bookmarking such as del.icio.us [3], photo sharing [4], research paper searching [2] and so forth. All of these sites use tagging for many purposes, but in addition to that, they focus on the social networking aspects of tagging to enhance the experience for end users. However, currently, tags are only used for tag searching-user profile matching and subsequent recommendations are yet to be implemented. As mentioned before, tags provide the clues as to why a user liked something. They are the who, what, when, where, and why of the user's reason for tagging something [6]. Because of this, as well as the similar use of social networking, social tagging systems provide an ideal choice for combination with CF systems.

Much of the research for tagging systems has been focused on user and tagging patterns. [10] uses a statistical approach towards deriving the emergent semantics of social tagging systems, namely del.icio.us. They use the Expectation Maximization algorithm (EM) to automatically cluster documents, users, and tags. They explored the optimal number of domain clusters as well as the number of iterative steps to get satisfactory cluster sizes and document classification. Lastly, they explored creating a personalized tag search engine based upon their findings.

\subsection{TCCF Website Recommendation System}

TCCF is an website recommendation algorithm which combines traditional CF systems and social tagging systems. The essential idea is that CF provides personalization, and tags provide clues of the reasons why users liked something. Unlike traditional CF models which use numeric ratings, the TCCF model uses tags for calculating user similarity and score prediction.

In a social bookmarking service, the act of bookmarking a website is a strong indicator of whether something is liked. Additionally, we also have tagging information. Usually, the user will use tags to describe the resource from the user's perspective, and in most cases this is the reason why they liked something. Thus, the key difference between traditional CF and TCCF: In addition to considering whether or not a user likes a resource, it also takes into account why users like something by using the tagging information .

In the TCCF Website Recommendation System, users bookmark websites they like using tags, and subsequently, they can easily retrieve their bookmarks by searching with tags. Once the the user has added enough bookmarks, the first step is finding similar users. This is then followed by calculating a score prediction for every website that the similar user has rated. The score prediction value is basically the level that the system thinks the user will like something-the higher score, the more the user should like the website. Both of these steps consider bookmarking as well as the attached tags when generating recommendation candidates. In this paper, we extend this method and describe it in section 3 .

\section{REASONABLE TAG-BASED COLLABORATIVE FILTERING FOR SOCIAL TAGGING SYSTEMS}

We now explain our recommendation algorithm and its use in our recommendation system. The basis of our system is a website bookmarking system similar to del.icio.us. A sample usage pattern would be as shown in figure 1 .

Here, an arrow indicates a user has bookmarked a page and the tags above the arrow indicate the tags used while bookmarking. User $A$ is bookmarking website 1 with the tags 'japanese' and 'dictionary'. Similarly, user $B$ is bookmarking website 2 with the tags 'apple' and 'news'. Based upon this type of social bookmarking system, our system generates effective recommendations based upon the user's bookmarking profile as well as their currently viewed page.

Since our recommendation algorithm is heavily based upon comparing websites using their topics, we first explain how we generate topic domain vectors.

\subsection{Generating Topic Domain Vectors}

In our previous iteration of our algorithm, similarity calculation between websites was based on the cosine of the website tag vectors. This tag vector was a feature vector where the parameters consisted of all the tags attached by users. For example, in figure 1, website 1's vector would have a value of one for 'japanese' and 'dictionary'. Website 2 would have values of two, two, one, one, and one for 'apple', 'news', 'games, 'reviews', and 'tech'. 


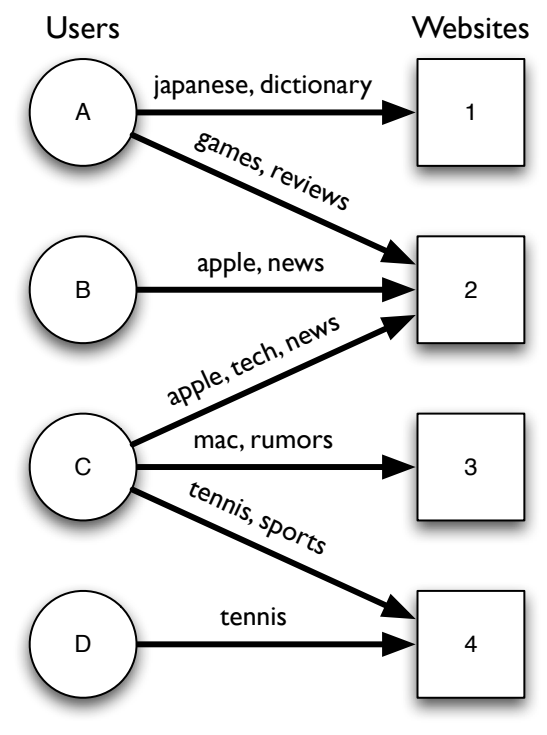

Figure 1: Website Bookmarking System Overview

This approach has the inherent problems that are associated with natural language processing. For example, vectors containing two synonyms, such as 'funny' or 'humourous', should be similar; however, when comparing only the base tag, these synonyms are considered as different terms, and thus, their vector cosine value will be low. To deal with this, we applied a similar approach to that of [10], by using the EM algorithm to cluster the websites into topic domains. Once this was done, we compared the websites based upon their topic vector rather than their tag vector.

Our data consists of three months worth of mining of del.icio.us RSS feeds. In total, we have over 100,000 users, 2.5 million webpages, 3.6 million bookmarks, and 870,000 distinct tags. After mining the data, we subsequently stemmed the tags using the well-known Porter stemming algorithm. Then, the number of unique stemmed tags was about 780,000. Over this data, we ran the EM algorithm for 50 iterations over 20,000 of the top bookmarked documents and the top 20,000 used tags and clustered them into 100 topic domains. The number of topic domains was chosen after a subjective comparison of topic separation they produced. Finding the optimal number of topic domains is described in [10] and is beyond the scope of our research.

Based on these topic domain clusters, website feature vectors were created for each document as shown in equation 1. We will from now refer to these as 'topic domain vectors' and abbreviate it as $D V$.

$$
D V_{k}=\left(d w_{1, k}, d w_{2, k}, d w_{3, k} \ldots, d w_{100, k}\right)
$$

Here, $d w_{j, k}$ is the topic domain weight of a website $k$ for a topic domain $j$. In other words, this is how strongly the document belongs in a given topic. This is calculated by summing the product of the conditional probability and tag term weight for all tags attached to website $k$. This is calculated as shown in equation 2 .

$$
d w_{j, k}=\sum_{i=0}^{n} P\left(t_{i} \mid D_{j}\right) \cdot t f_{i, k} \cdot i d f_{i}
$$

In this case, $n$ is the number of distinct tags attached to website $k$ by all users. $P\left(t_{i} \mid D_{j}\right)$ is the conditional probability of tag term $i$ (shown as $t_{i}$ ) given domain $j$ (shown as $\left.D_{j}\right) . t f_{i, k} \cdot i d f_{i}$ is as calculated by standard tf-idf, where $t f_{i, k}$ is the number of times any user bookmarked website $k$ with tag $i$ divided by the total number of times that the website is bookmarked. Similarly, the $i d f_{i}$ is determined by the total number of websites in the entire set divided by the number of websites bookmarked with tag $i$. So, instead of the website vector consisting of tag term counts, a website's domain vector parameters are now its topic domain weight value, i.e. how much the document belongs to that particular topic domain. An example calculation is shown in figure 2 .

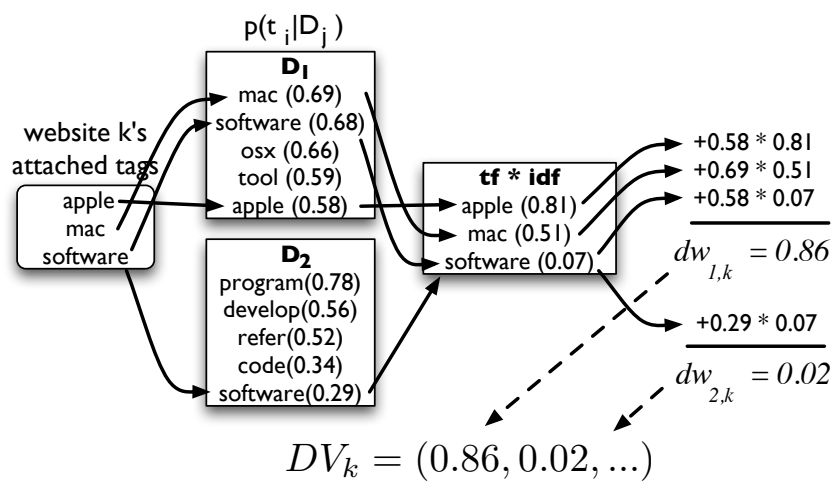

Figure 2: Calculation of $k=$ apple.com's website topic domain vector

In this case, apple.com has three tags attached by all users: 'apple', 'mac', and 'software'. Using example domain term probability values, we show the calculations for hypothetical domains 1 and 2 for a website $k$.

Similarly, a bookmark topic domain vector created from user $A$ bookmarking website $k, D V_{A \rightarrow k}$, would be calculated in the same fashion as shown in equation 3.

$$
D V_{A \rightarrow k}=\left(d w_{1, A \rightarrow k}, d w_{2, A \rightarrow k}, d w_{3, A \rightarrow k}, \ldots d w_{100, A \rightarrow k}\right)
$$

The only exception is that the domain tag weight only considers the tags that user $A$ used on website $k$ as shown in equation 4 .

$$
d w_{j, A \rightarrow k}=\sum_{i=0}^{n} P\left(t_{i} \mid D_{j}\right) \cdot i d f_{i, A \rightarrow k}
$$

Since a user can only apply atmost one of the same tag term to a bookmark, only the idf is used here.

\subsection{RCF Recommendations}

We now describe our Reasonable Tag-based Collaborative Filtering (RCF) algorithm. The process to generate recommendations follows these steps:

1. Finding similar users.

2. Finding recommendation candidates.

3. Providing live-updating recommendations based upon the user's current interest.

We now describe these steps. 


\subsubsection{Finding Similar Users}

We start with finding similar users. Similar to traditional collaborative filtering, it is based upon commonly liked items-in this case, commonly bookmarked websites. However, we also consider the used tags-which we assume to be the reason why a user liked a resource. For example, from our previously shown system in figure 1, suppose we want to find similar users for user $B$. It would be calculated as shown in figure 3 .

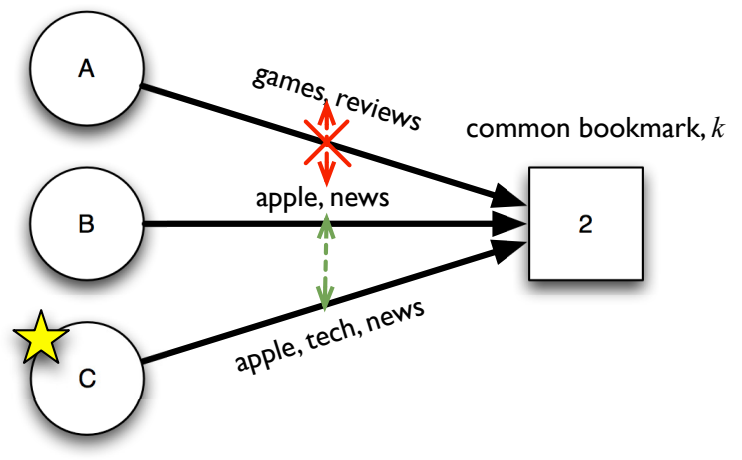

\section{Figure 3: Example User Similarity Calculation}

Since user $B$ and $C$ have bookmarked the same website with similar tags-indicated by the dotted arrow-their similarity score is higher. In this case, $C$ becomes a similar user, indicated by the star. On the other hand, users $B$ and $A$ have bookmarked the same site, but they do not use similar tags-indicated by the crossed-out dotted arrow-and thus, their similarity score is lower.

User similarity for a user $A$ and a user $B$ is calculated as shown in equation 5 .

$$
\begin{array}{r}
\operatorname{sim}_{r c f}(A, B)=\alpha \cdot \frac{1}{n} \sum_{1}^{n}\left\{\operatorname{sim}\left(D V_{A \rightarrow k}, D V_{B \rightarrow k}\right)\right\} \\
+(1-\alpha) \cdot \log _{2}(1+n)
\end{array}
$$

Here, user similarity is the average of the cosine of $A$ and $B$ 's domain vectors for each commonly bookmarked website, $k$. In other words, if they bookmarked the same websites with similar tags, we assume that they liked the website for the same reasons, and thus, they are similar users. The first half of the equation calculates the similarity between the topic domain vectors of each common bookmark for both users. The second half of the equation represents the number of common bookmarks and increases as the number of common bookmarks goes up.

Also, $n$ is the number of bookmarks that user $A$ and user $B$ have in common. $\alpha$ is the weight given to the topic domain vector comparison, which for our experiments was set to 0.9. Additionally, $\operatorname{sim}\left(D V_{A \rightarrow k}, D V_{B \rightarrow k}\right)$ is the cosine of user $A$ 's bookmark domain vector on website $k$ and user $B$ 's bookmark domain vector on website $k$ as shown in equation 6.

$$
\operatorname{sim}\left(D V_{A \rightarrow k}, D V_{B \rightarrow k}\right)=\frac{D V_{A \rightarrow k} \cdot D V_{A \rightarrow k}}{\left|D V_{A \rightarrow k}\right|\left|D V_{B \rightarrow k}\right|}
$$

Different from traditional CF, users must use similar tags on the common website in addition to having the common website liked. This is especially important when considering larger sites, such as Yahoo! or Slashdot, because they tend to cover many topics, and thus, users may like the same site for different reasons.

\subsubsection{Finding Recommendation Candidates}

Next, we find recommendation candidates by calculating score prediction-the score which represents how much the user should like a resource. Again, similar to traditional collaborative filtering, we recommend websites that the similar users liked, except that now we also additionally match the topic domain that the original user and the similar user have a common interest on. The goal being to recommend websites that match the topic that the two users commonly liked.

For example, in the system shown in figure 1, we will find recommendation candidates for user $B$ (user $B$ has a similar user $C$-shown in figure 4). User $B$ and $C$ both liked website 2 for the reason that the website is about apple technology news. Thus, we find websites that match this topic. In this case, similar user $C$ has two other bookmarks. Since only website 3's domain vector matches the commonly bookmarked website 2's domain vector-indicated by the dotted arrow-it has a high score prediction, and thus, becomes a recommendation candidate (denoted by a star). On the other hand, C's other bookmark, website 4, does not match the commonly bookmarked website's topic domain-marked by the crossed-out dotted line-and thus, its score prediction is lower.

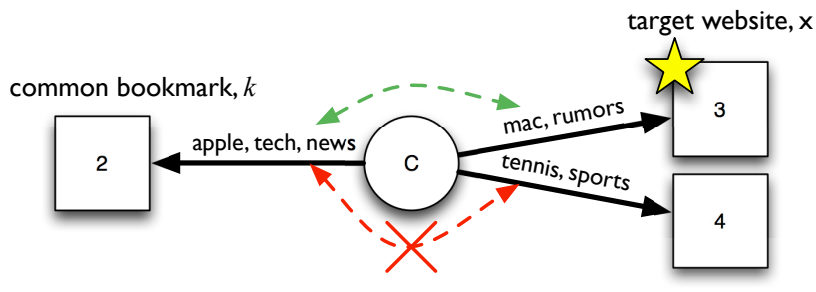

Figure 4: Example score prediction calculation

The score prediction algorithm is shown in equation 7 . Here, we are finding the score prediction for a target website $x$ for a user $A$. We first take all bookmarks from all similar users $S_{k}$ with a user similarity above a certain user similarity threshold-in our case, 0.75. We then compare each of those bookmark domain vector $D V_{S_{k} \rightarrow x}$ with the bookmark domain vector on the commonly bookmarked website $k, D V_{S_{k} \rightarrow k}$. We average these scores from all users and then those webpages with scores above a certain threshold0.50 in our tests-become recommendation candidates. Additionally, we record the association between each commonly bookmarked webpage $k$ and its generated recommendations. This is used in the final score calculation described in the following section.

Also, $\alpha$ is the weight given to the domain vector comparison, and in this case, it is 0.90 . The left side calculates the similarity between the topic domain vectors of the common bookmark and the target bookmark, and the right side of the equation represents the number of similar users that have bookmarked the target website $x$. 


$$
\operatorname{score~}_{\text {pred }}(A, x)=\alpha \cdot \frac{\sum_{k=1}^{n}\left\{\operatorname{sim}_{r c f}\left(A, S_{k}\right) \cdot \operatorname{sim}\left(D V_{S_{k} \rightarrow k}, D V_{S_{k} \rightarrow x}\right)\right\}}{\sum_{k=1}^{n}\left\{\operatorname{sim}_{r c f}\left(A, S_{k}\right)\right\}}+(1-\alpha) \cdot \log _{2}\left(1+\sum_{k=1}^{n}\left\{\operatorname{sim}_{r c f}\left(A, S_{k}\right)\right\}\right.
$$

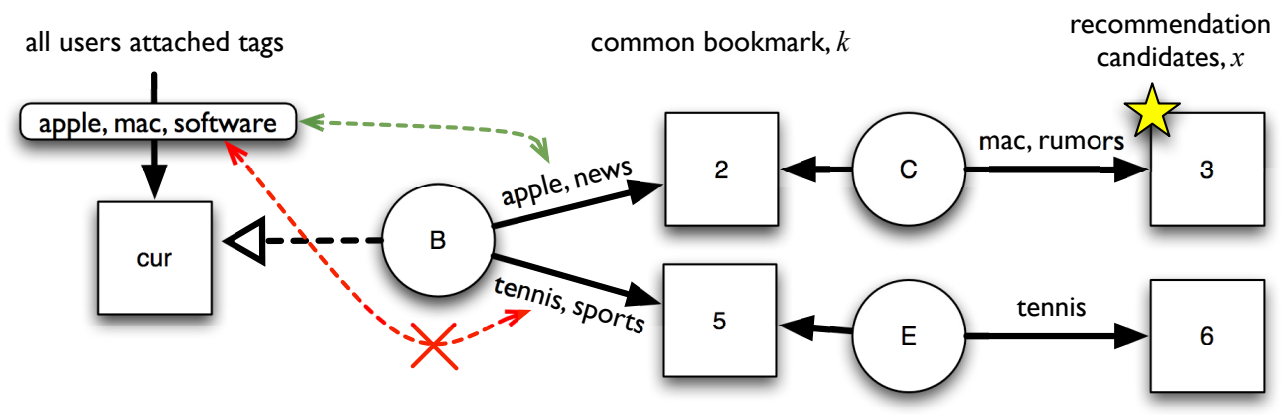

Figure 5: Example Final Score Calculation

\subsubsection{Providing Live-Updating Recommendations Based Upon User's Current Interest}

The last step in the process of generating recommendations is determining which of the recommendation candidates match the current interest. In our case, we assume the user's current interest to be the topic of the website they are presently viewing. We then generate the topic domain vector for the currently viewed website by using the tags from all user's bookmarks from our mined data set. For example, in the system shown in figure 1, we provide recommendations for user $B$ as shown in figure 5 .

Here, $B$ is looking at a site which has the tags 'apple', 'mac', and 'software' attached to it by bookmarks from all users. $B$ also has two recommendation candidates, website 3 and 6 (6 is an arbitary webpage not shown in the example system), which were generated from section 3.2.2. Website 3 has been associated with the commonly bookmarked website 2 , and website 6 has been associated with website 5 . We then compare the user's currently viewed webpage's domain vector to all of $B$ 's bookmarks. If we find one that is above a certain threshold, we take the recommendation candidates that are attached to it and calculate its final score as shown in equation 8. All websites above a certain threshold are then recommended to the end user.

In this case, since $B$ 's bookmark domain vector matches the current website's domain vector, its attached website, 3 , is used for final score calculation. If its score is above a certain threshold, it is recommended. $B$ also has an arbitrary recommendation candidate, website 6 . However, since $B$ 's bookmark on website 5 does not match the currently viewed website, its associated recommendation candidate is not recommended at this time. If the current website were to change to something about sports or tennis, website 6 would be recommended if its final score was above the selected threshold.

In equation 8 , user $A$ is viewing webpage $c u r, k$ is the commonly bookmarked website, and $x$ is a recommendation candidate. Lastly, $\beta$ is the weight given to the part of the equation which determines how similar the recommendation candidate should be to the current page. For our experiments, this was set to 1 . All resultant final scores higher than a threshold of 0.50 are shown to the user in the descending order of the score.

\subsection{Live-Updating Website Recommendation Prototype System}

Using our RCF algorithm, we created a prototype recommendation system. It was created to provide live-updating website recommendations that automatically update based on the page that they are currently viewing. Thus, it was designed to be viewable during the user's normal browsing activity. It is currently implemented as a Firefox sidebar plugin. The recommendation interface is shown in figure 6. While the user is browsing, the recommendations are updated whenever the viewed website changes, thereby providing recommendations relevant to the user's current interest. Here, the generated recommendations are shown in the sidebar on the left of the image. If they feel the recommendations are useful, they can click on it and the browser will be redirected to the recommended website.

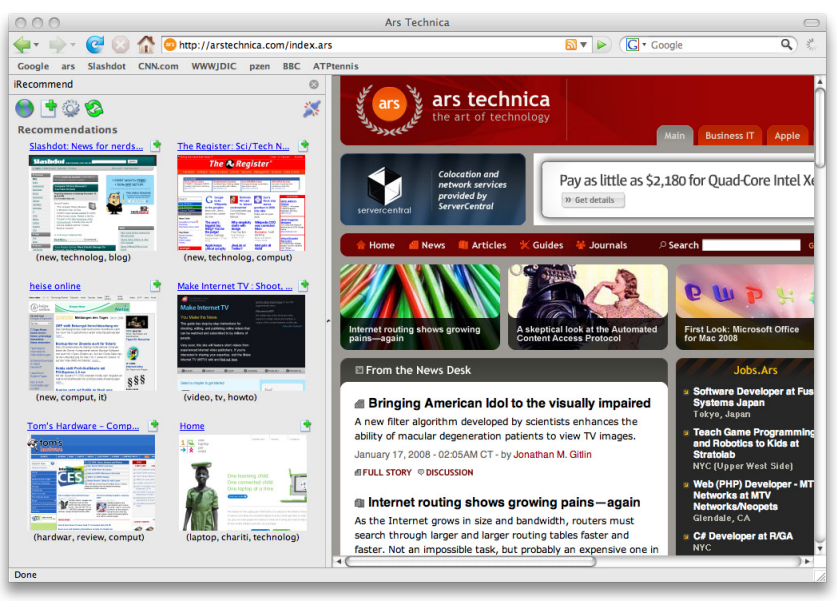

Figure 6: System Interface 


$$
\operatorname{score}_{\text {final }}(A, \operatorname{cur}, x)=\operatorname{sim}\left(D V_{\text {cur }}, D V_{A \rightarrow k}\right) \cdot \operatorname{score}_{\text {pred }}(D V, x) \cdot \operatorname{sim}\left(D V_{\text {cur }}, D V_{A \rightarrow x}\right)^{\beta}
$$

\section{EXPERIMENT}

As mentioned before in section 3.1, we mined del.icio.us RSS feeds for three months. In total, we retrieved over 100,000 users, 2.5 million webpages, 3.6 million bookmarks, and 870,000 distinct tags. After mining this data, we performed user testing with our algorithm against other popular search methods to gauge the effectiveness of the algorithm as well as observe how users react to generated recommendations. We tested our method as described in the previous section as well as three other different methods:

- topic - This method takes the top five topic domains from the currently viewed webpage and then finds all the bookmarks from the database with high values for those topic domains. All websites above a cosine value of 0.75 are then ordered by that value and shown to the user

- tag-bookmark-count (tbc) - This method takes the top three stemmed tags from the currently viewed webpage and then pulls all bookmarks from the database with these tags. The results are then ranked by the number of bookmarks in descending order.

- cf-topic - All bookmarks of all users with common bookmarks with the current user were stored as recommendation candidates. Then we take the currently viewed website's domain vector and those stored bookmarks' domain vectors and calculate the cosine. They are then ranked by cosine value. This has similarity to our method, but it does not consider tagging when calculating the score predictions of the recommendation candidates

Using the Firefox plugin, users were asked to create a profile by bookmarking twenty or more websites using tags. They were free to use any webpage they liked with any tags they felt were appropriate. After their profiles were created, similar users as well as recommendation candidates were calculated as shown in section 3.2.1 and 3.2.2. This step occurred beforehand and not while the user was browsing. The last step of selecting recommendation candidates to display (using the final score as shown in section 3.2.3) was done in real-time while the user was browsing.

Each user followed this recommendation evaluation procedure for fifteen or more websites of their choosing:

1. The user opens a website in firefox.

2. Based upon the website, the system generates up to six recommendations and displays them in the sidebar plugin. This interface was shown previously in 6 . The system randomly chooses one of the previously described algorithm. In the case of our algorithm, it calculates the final score as shown in section 3.2.3. It does so by comparing the domain tag vector of the currently viewed site and the domain tag vector associated with each recommendation candidate. It would display the top 6 results that were above the final score threshold of 0.5 . The other methods would do their respective procedure using the current page's tags.
3. Users would then look at the recommended website's screenshot thumbnail, top tags, and title. They could choose to click and view it or not.

4. If they did click on a recommendation, the browser would open the website. Additionally, rating buttons would appear at the bottom of the sidebar. Here, they were asked to evaluate the website as either 'Good', 'Fair', or 'Poor' in terms of the following two conditions: First, how interesting the page was. Second, whether the website was related to the page that they were currently viewing.

The experiment was done with eleven student volunteers. We now explain our results of our user testing.

\subsection{Results}

\subsubsection{How many bookmarks are needed before rec- ommendations can be made?}

One of the difficulties in CF systems is getting the user to evaluate and rate enough items. If there are not enough items rated, the system cannot confidently find similar users, and therefore cannot find accurate recommendations as well. Thus, we explore how many bookmarks are necessary before the system can produce recommendations.

Figure 7(a) shows a user's total number of bookmarks versus the ratio of users with at least one common bookmark with another user. For example, about $40 \%$ of the users with one to five total bookmarks have a bookmark in common with another user. Similarly, $80 \%$ of users with eleven to fifteen bookmarks have at least one common bookmark with another user.

Next, figure 7(b) shows a user's total number of bookmarks versus the ratio of users with at least one recommendation candidate with a score prediction above 0.50 (explained in section 3.2.2). At the sixteen to twenty bookmark range, a little more than $80 \%$ of the users have recommendation candidates. After this point, the rate of increase in the proportion of users with recommendations decreases.

Even with a few bookmarks, the system is able to generate recommendations for the candidates. It is also largely dependent on which website you bookmark and whether that website has been bookmarked by another user.

\subsubsection{How often are recommendation candidates be- ing shown?}

We have shown that our algorithm can generate recommendation candidates with just a few bookmarks. Next, we take a look at how often these candidates are being shown to the user in the final step of providing live-updating recommendations. Thus, in table 1 , we have listed the number of recommendations generated per times requested.

The first column shows the number of recommendation requests. The second shows the number of recommendations that were returned in total. The third column shows the average number of recommendations per request. For each request, there was a maximum of six recommendations returned. Last, there is the adjusted rate, which is the approximate ratio of the number of times that each method 


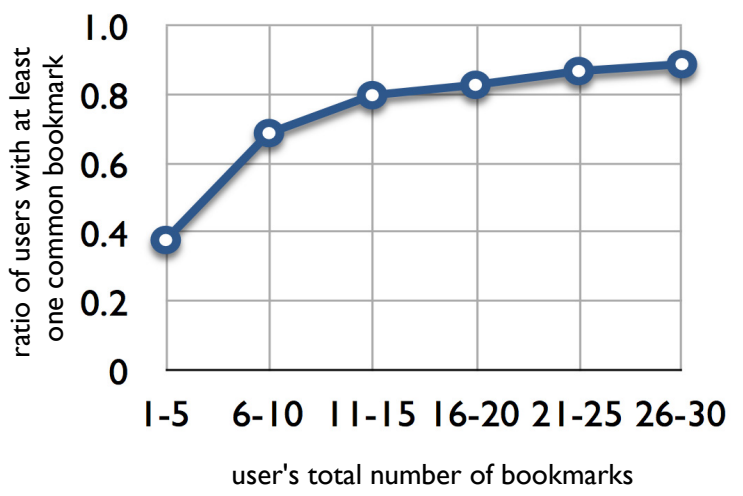

(a) User's total number of bookmarks vs. ratio of users with at least one common bookmark

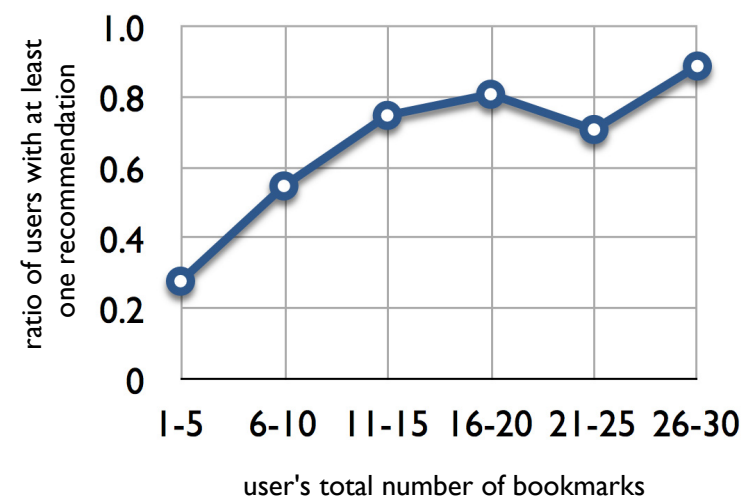

(b) User's total number of bookmarks vs. ratio of users at least one recommendation

Figure 7: User's Total Number of Bookmarks vs ...

\begin{tabular}{|c|cccc|}
\hline & $\begin{array}{c}\text { num. } \\
\text { req. }\end{array}$ & $\begin{array}{c}\text { num. } \\
\text { recom. }\end{array}$ & $\begin{array}{c}\text { recom. } \\
\text { per req. }\end{array}$ & $\begin{array}{c}\text { adj. recom. } \\
\text { rate }\end{array}$ \\
\hline topic & 173 & 645 & 3.73 & 0.621 \\
tbc & 172 & 641 & 3.73 & 0.621 \\
cf-topic & 169 & 628 & 3.72 & 0.619 \\
rcf & 170 & 358 & 2.11 & 0.351 \\
\hline
\end{tabular}

Table 1: Amount of Recommendations Generated

had at least one recommendation for a request. For topic, tbc, cf-topic, this came out to roughly $2 / 3$ of the time. For $\mathrm{RCF}$, this came out to a little more than $1 / 3$ of the time.

Our system evenly spread the requests over the methods. Due to the specific nature of our algorithm, our method tended to generate less than the other methods. Additionally, our mined data set was relatively small.

\subsubsection{How effective are the recommendations?}

Now, we examine how effective each method was for recommendation. Figure 8 shows the precision of each of the recommendation methods.

Precision is defined as the number of 'Good' ratings over the total number of ratings. Again, each user was asked to

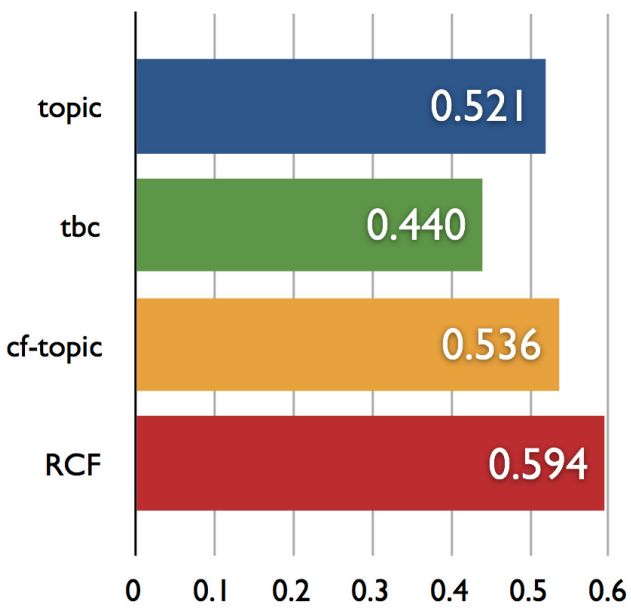

Figure 8: Precision (Good / Total)

rate every recommendation they visited, with either 'Good', 'Fair', 'Poor'. Here, each method's precision is shown. As can be seen here, our method RCF provides the highest level among all the recommendation methods. It is followed by cf-topic, topic, and then finally tbc.

Additionally, we take a look at the average ratings for the top three recommendations per request. This is shown in figure 9. Here, we have the average rating for the top

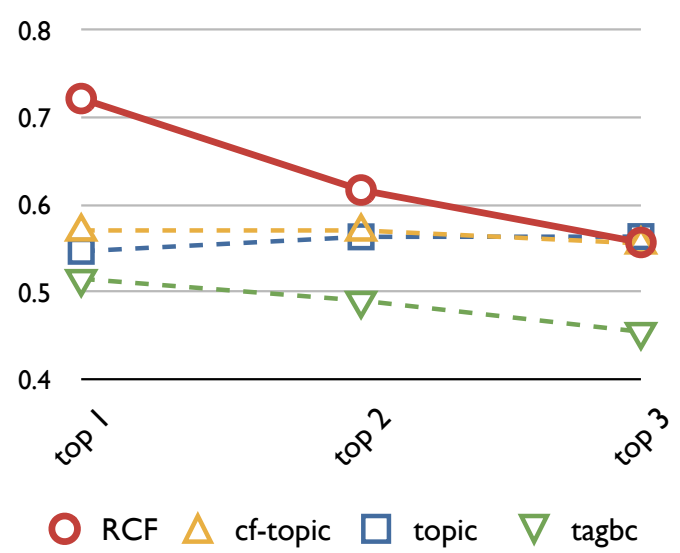

Figure 9: Average Score of the Top Results per Request

listed result, followed by the top two, and finally top three results per recommendation request. The ratings were given the following values: 'Good' $=1$, 'Fair' $=0.5$, and 'Poor' $=0$. Again, within the recommender interface, up to six recommendations are shown in the sidebar, ordered from the highest final score to the lowest. RCF has the highest precision among all the methods when considering only the top results per request. 


\subsection{Results Discussion}

We first showed that our algorithm is capable of generating recommendations even when only a few websites have been bookmarked. This is an advantage over traditional CF systems, which require more input for it to be effective.

In terms of amount of recommendations generated, it can be seen that our recommendation method did not generate as many results as the other methods. Since our method is more specific, it resulted in a lower number of recommendation candidates. Additionally, this can be attributed to the fact that del.icio.us is largely used by technology-savvy people-therefore, much of the bookmarking data is for websites that are largely technology-oriented. Since some of the testers did not bookmark websites in a technology-related topic domain, our system could not find any recommendation candidates with a final score above the threshold. In the future, when social bookmarking becomes used by a wider audience, the variety of data should expand and allow for recommendations in more domains.

Looking at the precision data, our RCF method provides the most relevant recommendations to the user in terms of the precision of the recommendation results. By taking into account tagging information, RCF is able to more effectively filter out the appropriate results. Additionally, it can recommend websites that are related to what the user is currently viewing in real-time.

Topic searching was the third most effective method in our testing. Its algorithm is entirely based on the tagging information from the current page. It does not use the user's bookmarking profile in any way.

tbc ended up last in its precision value. It takes website popularity into consideration as its ranking method orders by bookmark count. However, since tbc does not consider the user's bookmarking profile, it is unable to provide personalized recommendations.

Lastly, cf-topic came up second place. It is a basic implementation of collaborative filtering: it considers the user's profile to some degree. However, it does not utilize the tagging information when performing collaborative filtering. Since social bookmarking systems do not have numeric ratings at all, traditional CF is very ineffective. A user can only indicate liking a website with a bookmark or not-there is no rating in between, nor are there negative ratings.

Regarding the top results' values, RCF provided a higher precision when considering the top results. This is important in recommendation systems as there is a limit on how many recommendations you can provide to the end user. Being so, it is more important to provide high precision over high recall.

\section{CONCLUSIONS AND FUTURE WORK}

We have described our Reasonable Tag-Based Collaborative Filtering method for use in social tagging systems. Additionally, we have described its place in our live-updating website recommendation system. Through our user testing, we have shown that live-updating implicit recommendation is possible. We found it to be more effective than other tested methods, and additionally provided higher ratings for the top results per recommendation request.

Overall, the system we have proposed covers a large, vast domain-making the task of providing recommendations difficult. Thus, there are still many unexplored factors effect- ing the results. However, we believe it is an effective method that can be employed in all sorts of online tagging systems. Social tagging systems and CF rely heavily on the people using these systems, so combining them as done in our method seems like the next logical step. Doing so provides relevant recommendations in which the user has confidence in the credibility of the source. Social tagging systems would benefit greatly from an effective recommendation system.

In the future, we plan to further refine the recommendation method. We are evaluating what the optimal threshold is for the final score. Next, we also intend to add relevance feedback into our method. Currently, it is based solely on bookmarks. However, adding positive or negative feedback of the recommendations generated would allow the system to provide better recommendations later on. Lastly, we plan to add recommendation grouping, where the results are sorted and grouped by topic. This way, the users can have a better idea of what the website contains before they choose to view it.

\section{Acknowledgments}

This work was supported in part by the National Institute of Information and Communications Technology, MEXT (Grantin- Aid for Scientific Research on Priority Areas \#19024058), and MEXT (Grant-in-Aid for Young Scientists (B) \#20700089).

\section{REFERENCES}

[1] Amazon.com. http://www.amazon.com/.

[2] Citeulike. http://www.citeulike.org/.

[3] del.icio.us. http://del.icio.us/.

[4] Flickr. http://www.flickr.com/.

[5] movielens. http://www.movielens.umn.edu/.

[6] S. Golder and B. Huberman. The structure of collaborative tagging systems.

[7] R. Nakamoto, S. Nakajima, J. Miyazaki, and H. K. S. Uemura. Evaluation of tag-based contextual collaborative filtering effectiveness in website recommendation. Technical Report 131, IEICE, 2007.

[8] R. Nakamoto, S. Nakajima, J. Miyazaki, and S. Uemura. Tag-based contextual collaborative filtering. In 18th IEICE Data Engineering Workshop, 2007.

[9] P. Resnick, N. Iacovou, M. Suchak, P. Bergstorm, and J. Riedl. GroupLens: An Open Architecture for Collaborative Filtering of Netnews. In Proceedings of ACM 1994 Conference on Computer Supported Cooperative Work, pages 175-186, Chapel Hill, North Carolina, 1994. ACM.

[10] X. Wu, L. Zhang, and Y. Yu. Exploring social annotations for the semantic web. In $W W W$ '06: Proceedings of the 15th international conference on World Wide Web, pages 417-426, New York, NY, USA, 2006. ACM. 